\title{
LOS ESTUDIOS DE ASIA ORIENTAL EN ESPAÑA: LOS ITINERARIOS SOBRE CHINA Y SU LENGUA EN LOS GRADOS UNIVERSITARIOS
}

\author{
EASTERN ASIA STUDIES IN SPAIN: STUDY PLANS \\ ABOUT CHINA AND ITS LANGUAGE \\ IN UNIVERSITY DEGREES
}

María Querol Batallera*

Fechas de recepción y aceptación: 8 de junio de 2020 y 29 de junio de 2020

DOI: https://doi.org/10.46583/edetania_2020.58.686

\begin{abstract}
Resumen: En este trabajo se muestra un panorama esencialmente sincrónico de los estudios que sobre el chino y China se ofertan en el nivel de grado universitario en España. Para ello se describe la oferta académica existente tanto en grados específicos como en grados el ámbito de humanidades y ciencias sociales. Con tal acción se pretende esbozar no solo su génesis o localización geográfica, sino también su estructuración y organización según consta en los planes de estudios y guías docentes que las universidades hacen públicos en sus respectivas páginas webs. Una reflexión de este tipo es imprescindible para valorar si la oferta académica se corresponde con las demandas de la sociedad, si su organización permite optimizar los recursos existentes o si existen demandas o necesidades por atender.
\end{abstract}

Palabras clave: español, chino, universidad, grado, plan de estudios.

Abstract: The aim of this work is to present a synchronous overview of studies on the Chinese language and China offered at the university degree-level in Spain. To do so, the existing academic offerings in specific degrees and in degrees in the fields of the humanities and social sciences have been researched. This work outlines not only the origin or geographical Mártir.

a Facultad de Magisterio y Ciencias de la Educación. Universidad Católica de Valencia San Vicente

* Correspondencia: Universidad Católica de Valencia San Vicente Mártir. Facultad de Magisterio y Ciencias de la Educación. Calle Sagrado Corazón, 5. 46110 (Godella), Valencia. España.

E-mail: maria.querol@ucv.es 
location of such studies, but also their structure and organisation as stated in the curricula and teaching guides that universities publish on their websites. A reflection of this type is essential to assess whether the academic offerings correspond to the demands of society and whether organisations could optimise existing resources or address unmet demands or needs.

Keywords: Chinese, China, university, degree, study plan.

\section{INTRODUCCIÓN}

El desarrollo sociopolítico y económico de China en las últimas décadas ha hecho evidente a la sociedad española, y también a los responsables políticos y académicos, la necesidad de contar con expertos en lengua y cultura china, pero también en diversos aspectos relacionados con su organización social y política. A tal efecto, se posibilitó en los inicios del siglo XXI la implantación en algunas universidades españolas del grado en Estudios de Asia Oriental, el grado en Lenguas y Literaturas Modernas con itinerario específico sobre el chino y China, y también diversos másteres vinculados a esta área de conocimiento.

Dicho esto, en este trabajo se realiza un estudio exploratorio que trata de mostrar un panorama esencialmente sincrónico de los estudios que sobre el chino $^{1}$ y China se ofertan en el nivel de grado universitario en España. Con esta acción se trata de dar a conocer la oferta académica que con respecto a la lengua, cultura y sociedad china se realiza tanto en grados específicos como en otros grados del área de humanidades o del área de ciencias sociales, cuyos egresados, en el ejercicio de sus competencias profesionales, pudieran requerir de cierto grado de competencia comunicativa en esta lengua, o en las estructuras sociales, económicas, políticas o culturales de las sociedades que la hablan. Una reflexión de este tipo es imprescindible para valorar si la oferta académica se corresponde con las demandas de la sociedad, si su or-

${ }^{1}$ En este trabajo la palabra chino o lengua china se usa para denotar la variedad estándar de la lengua china, el 普通话 (putonghua). China no es una comunidad homogénea en términos lingüísticos; así, en muchos lugares el puntonghua convive con otras variedades de la lengua china, que, en términos oficiales, tienen la consideración de dialectos. Además, en muchas provincias el chino convive con las lenguas de las llamadas nacionalidades minoritarias, que, a diferencia de lo que ocurre con los dialectos, sí tienen estatuto de oficialidad en las regiones donde tradicionalmente se asientan las nacionalidades que las hablan (Prosser, 2007). 
ganización permite optimizar los recursos existentes o si existen demandas o necesidades por atender.

Dado que la Agencia Nacional de Evaluación de la Calidad y Acreditación (ANECA) es el organismo encargado de verificar y acreditar los títulos oficiales que imparten las universidades españolas, así como sus respectivos planes de estudio, en este trabajo se recurre a su base de datos para conocer qué titulaciones se ofertan y en qué universidades. Por otra parte, para alcanzar los fines propuestos en este trabajo es necesaria la revisión de los planes de estudios y guías docentes que las universidades hacen públicos mediante sus respectivas páginas webs y que han recibido la acreditación positiva por parte de ANECA. ${ }^{2}$

\section{AnteCEDENTES}

Las revisiones que realizan López-Álvarez (1978) y Lu (2005) coinciden en situar el inicio del interés de chinohablantes e hispanohablantes por el español y el chino, respectivamente, en torno al siglo XVI. En gran medida este fue consecuencia de las necesidades e intereses generados en las rutas comerciales establecidas entre el sur de China y las colonias españolas en América, y a través de ellas con España (Baltar, 1993, p. 94).

López-Álvarez (1978) mostraba también cómo fueron los franciscanos y dominicos españoles quienes, en el ámbito occidental, inauguraron los estudios del idioma, pensamiento, costumbres e historia de China. Inicialmente desde la colonia española de Manila, más tarde desde la embajada establecida en la provincia de 福建 (Fujian) y, finalmente, desde la Universidad de Santo Tomás (Filipinas). Así, Juan Cobo realizó la primera traducción de un libro chino a una lengua occidental y Martín de Rada, considerado actualmente como el primer sinólogo de Occidente, escribió el primer tratado sobre el chino en una lengua occidental (Querol, 2010).

La supresión de los viajes del Galeón de Manila, también llamado Nao de China, la pérdida de las colonias americanas y asiáticas y el resto de avatares

\footnotetext{
${ }^{2}$ En este trabajo se utilizará información hecha pública a través de las respectivas webs para el curso 2019-20.
} 
sociopolíticos en los que se vieron inmersas China y España durante los siglos XIX y XX minaron considerablemente el interés en la enseñanza y aprendizaje de las respectivas lenguas. En torno a los años cincuenta y sesenta, a la par que se intensificaban las relaciones entre China y los países latinoamericanos, comenzaron a implantarse los estudios de licenciatura de español, a partir de 1979 también los de posgrado y más recientemente los estudios de doctorado. En España, sin embargo, aún hoy en las universidades españolas no existen departamentos de chino. Los estudios de o sobre esta lengua se inscriben en el área de conocimiento de Asia Oriental y esta está adscrita, dependiendo de las universidades, al departamento de Traducción e Interpretación, de Lingüística o de Lenguas Modernas. Además, no fue hasta casi la década de los noventa cuando las universidades españolas comenzaron a ofertar cursos relacionados con algún aspecto de la cultura china, su historia, su literatura, su lengua o su pensamiento filosófico. La Universidad Autónoma de Madrid (UAM), la Universidad Autónoma de Barcelona (UAB) y la Universidad de Granada (UGR) fueron pioneras en este aspecto, así como también en ofrecer la posibilidad de cursar chino como opción C en la licenciatura de Traducción e Interpretación. No obstante, hubo que esperar hasta el curso 2003/2004 para que se implantara una licenciatura de $2 .^{\circ}$ ciclo con competencias específicas sobre la región de Asia Oriental, y los primeros egresados en España en Estudios de Asia Oriental iniciaron su formación en el curso 2009-2010 (tabla 1).

TABLA 1

Grados implementados en España vinculados al área de Asia Oriental o con itinerario especifico sobre China o el chino

\begin{tabular}{|l|l|c|}
\hline \multicolumn{1}{|c|}{ Universidad } & \multicolumn{1}{|c|}{ Nombre de la titulación } & \multicolumn{1}{c|}{$\begin{array}{c}\text { Año de } \\
\text { inicio }\end{array}$} \\
\hline $\begin{array}{l}\text { Universidad Autónoma de Madrid } \\
\text { (UAM) }\end{array}$ & $\begin{array}{l}\text { Estudios de Asia y África: Árabe, Chino y } \\
\text { Japonés }\end{array}$ & $2009-2010$ \\
\hline $\begin{array}{l}\text { Universidad Autónoma de Barcelona } \\
\text { (UAB ) }\end{array}$ & Estudios de Asia Oriental & $2009-2010$ \\
\hline $\begin{array}{l}\text { Universidad de Sevilla (US) y } \\
\text { Universidad de Málaga (UM) }\end{array}$ & Estudios de Asia Oriental & $2011-2012$ \\
\hline Universidad de Salamanca (USAL) & Estudios de Asia Oriental & $2015-2016$ \\
\hline
\end{tabular}




\begin{tabular}{|l|l|c|}
\hline \multicolumn{1}{|c|}{ Universidad } & \multicolumn{1}{|c|}{ Nombre de la titulación } & \multicolumn{1}{c|}{$\begin{array}{c}\text { Año de } \\
\text { inicio }\end{array}$} \\
\hline Universidad de Granada (UGR) & $\begin{array}{l}\text { Lenguas y Literaturas Modernas, Maior en } \\
\text { chino }\end{array}$ & 2010-2011 \\
\hline $\begin{array}{l}\text { Universidad Autónoma de Barcelona } \\
\text { (UAB) }\end{array}$ & $\begin{array}{l}\text { Estudios de Español y de Chino: Lengua, } \\
\text { Literatura y Cultura }\end{array}$ & $2020-2021$ \\
\hline
\end{tabular}

Fuente: ANECA.

Es también en los últimos años del pasado siglo y en los inicios del siglo XXI cuando se iniciaron de forma oficial los estudios de posgrado, doctorado o máster con competencias específicas sobre el chino o China (tabla 2).

TABLA 2

Másteres implementados en España con itinerario especifico sobre China o el chino

\begin{tabular}{|l|l|}
\hline \multicolumn{1}{|c|}{ Universidad } & \multicolumn{1}{c|}{ Nombre de la titulación } \\
\hline $\begin{array}{l}\text { Universidad Autónoma de } \\
\text { Madrid (UAM) }\end{array}$ & Máster Universitario en Estudios de Asia Oriental \\
\hline $\begin{array}{l}\text { Universidad de Alcalá de } \\
\text { Henares (UAH) }\end{array}$ & $\begin{array}{l}\text { Máster Universitario en Comunicación Intercultural, } \\
\text { Interpretación y Traducción en los Servicios Públicos }\end{array}$ \\
\hline $\begin{array}{l}\text { Universidad de Granada } \\
\text { (UGR) }\end{array}$ & Máster Universitario en Estudios de Asia Oriental \\
\hline $\begin{array}{l}\text { Universidad Autónoma de } \\
\text { Barcelona (UAB) }\end{array}$ & $\begin{array}{l}\text { Máster Universitario en Investigación en Asia Oriental } \\
\text { Contemporánea }\end{array}$ \\
\hline $\begin{array}{l}\text { Universitat Pompeu Fabra } \\
\text { (UPF) }\end{array}$ & Máster Universitario en Estudios Chinos \\
\hline $\begin{array}{l}\text { Universitat Pompeu Fabra } \\
\text { (UPF) }\end{array}$ & $\begin{array}{l}\text { Máster Universitario en Traducción Entre Lenguas Globales: } \\
\text { Chino-español }\end{array}$ \\
\hline $\begin{array}{l}\text { Universitat Oberta de } \\
\text { Catalunya (UOC) }\end{array}$ & $\begin{array}{l}\text { Máster Universitario en Estudios de China y Japón: Mundo } \\
\text { Contemporáneo }\end{array}$ \\
\hline $\begin{array}{l}\text { Universidad Autónoma de } \\
\text { Barcelona (UAB) }\end{array}$ & Máster oficial de didáctica de chino como lengua extranjera \\
\hline
\end{tabular}

Fuente: ANECA. 
Supone un notable avance, sí, pero no debe olvidarse que en muchos de los países del entorno europeo, EE. UU., Canadá o Australia los estudios sobre Asia Oriental y sus lenguas cuentan ya con una larga y consolidada tradición (Ministerio de Asuntos Exteriores, 2002; Fisac, 2004; Rovira i Esteva 2006; Querol, 2010; Wang, 2010).

\section{LA SITUACIÓN ACTUAL}

\subsection{Estudios de lengua china en grados especificos}

En España la remodelación de los planes de estudio que ha supuesto la implantación del Plan Bolonia ha brindado una magnífica oportunidad a las universidades para darle mayor presencia al chino y a todo lo que tiene que ver con la cultura y sociedad china. Con respecto a esto, es preciso reseñar los siguientes hitos:

La anterior licenciatura de $2 .^{\circ}$ ciclo se ha convertido en un grado, un hecho que, lógicamente, permite mejorar notablemente la formación y especialización de sus egresados. Estos estudios duran 4 años y los 240 ECTS se distribuyen en 60 ECTS de asignaturas de formación básica, 21 ECTS de obligatorias, 153 de optativas (a través de las cuales se configuran las menciones) y 6 ECTS del trabajo fin de grado.

Inicialmente se propuso la denominación del grado como Lenguas y Culturas de Asia y África y, en este sentido, ANECA (2006, p. 435) indicaba que la denominación Asia y África es más completa y precisa que la denominación Lenguas Orientales. Sin embargo, las universidades no han optado por aquella dominación, probablemente porque las competencias exigidas en dicha titulación ni se circunscriben en todos los casos a aquellas áreas geográficas, ni se limitan al ámbito de la filología. Solo la UAM ha mantenido la denominación Estudios en Asia y África, no en vano es la única que incluye un itinerario específico para la región geográfica árabe e islámica.

Los Estudios de Asia Oriental, hoy grado en Estudios de Asia Oriental (EAO, en adelante) o Estudios en Asia y África (EAA, en adelante) nacieron con el propósito de capacitar al alumno para desenvolverse profesionalmente en dichas regiones, y no con el objetivo específico de formar a profesionales 
en el dominio de la lengua (Ministerio de Asuntos Exteriores, 2002, pp. 443446). Se consideró que la demanda profesional implicaba una formación de carácter interdisciplinar y no únicamente filológica; y, en consecuencia, se diseñó un plan de estudios que comprendiera materias tanto del ámbito de las humanidades como de las ciencias sociales, y que incluyera tanto aspectos tradicionales como contemporáneos de la región. Esto supuso la activación de asignaturas troncales y obligatorias, ahora llamadas de formación básica (FB) y obligatoria $(\mathrm{OB})$, referidas a la lengua, pero el plan de estudios de la titulación no se articula en torno a estas. La mayoría de las universidades optó por otorgar a dichas materias de lengua un porcentaje obligatorio en torno al 20 $\%$ del total. No obstante, esta formación en las materias de lengua de carácter prescriptivo puede ampliarse cursando las diferentes asignaturas optativas que se ofertan (tabla 3 ).

TABLA 3

Porcentaje de materias de lengua china en los grados de EAA/EAO y LLM, maior Chino

\begin{tabular}{|l|l|c|}
\hline \multicolumn{1}{|c|}{ Grado } & Universidad & $\begin{array}{c}\text { Porcentaje de ECTS vinculados } \\
\text { al dominio de la lengua china }\end{array}$ \\
\hline EAA & UAM & $22,5-25 \%$ \\
\hline EAO & UAB & $17,5-30 \%$ \\
\hline EAO & US /UM & $20-25 \%$ \\
\hline EAO & USAL & $32,5 \%$ \\
\hline $\begin{array}{l}\text { Lenguas y Literaturas Modernas, } \\
\text { maior en chino }\end{array}$ & UGR & $20-30 \%$ \\
\hline
\end{tabular}

Fuente: elaboración propia.

Por primera vez en la realidad universitaria española el grado en Lenguas y Literaturas Modernas, maior en chino, dotó a esta lengua de un ámbito de estudio con entidad propia y, a diferencia del carácter interdisciplinar del grado en EAO o EAA, se circunscribe únicamente en el ámbito de la Filología. El grado de la UGR sería, por tanto, el que más se asemejara a la licenciatura de Español que cursan nuestros homólogos chinos; no obstante, en el plan de estudios de la UGR se observa que el porcentaje de créditos establecidos para el 
estudio de la lengua es semejante al de los grados de EAO o EAA. Pese a todo ello, el porcentaje de las materias de lengua es significativamente inferior al de sus homólogos en China, que se sitúa en torno al 70 \% (Querol, 2014).

Por otra parte, y a diferencia de lo que ocurre en el grado de Español en China, tanto en el grado en Lenguas y Literaturas Modernas, maior en chino, como en el grado en EAO o EAA en las asignaturas sobre lengua china las destrezas no se estudian de forma independiente. Desde el primer nivel los objetivos y resultados de aprendizaje establecidos denotan que el alumno debe aplicar las competencias aprendidas mostrando sus habilidades comunicativas en las diferentes destrezas. De igual forma, los contenidos sistemáticamente hacen referencia a los aspectos fonológicos, léxico-morfológicos, gramaticales, socioculturales y funcionales de la lengua. No obstante, existen algunas excepciones. Por ejemplo, en algunas titulaciones se dedican asignaturas que permiten al alumno iniciarse en una formación especializada en traducción (Traducción de textos de la primera lengua: chino, UAM), en el uso de la lengua para fines específicos (Lengua para fines especificos: chino, UAB; Mediación intercultural en lengua china, UGR), en la escritura y la caligrafía (UGR, USAL) o en la lengua clásica (UAB).

Por otra parte, el plan Bolonia dota, o mantiene, la autonomía de las universidades para decidir sobre el perfil de sus egresados mediante, por ejemplo, la implantación de menciones o determinadas asignaturas obligatorias $\mathrm{u}$ optativas. En este sentido, se observa cómo, antes de que las administraciones subrayaran públicamente la necesidad de "ordenar" el sistema universitario español, los responsables de diseñar los diferentes planes de estudio sí debieron de percibir la necesidad de dotar a los diferentes Estudios de Asia Oriental de un perfil propio y singular en cada centro. Dada la relevancia y el interés que despierta China, parece lógico que todas las universidades que han implantado el grado en EAO o EAA hayan implementado también una mención específica sobre esta. Sin embargo, la posibilidad de cursar otras menciones u otras asignaturas optativas dota a estos grados de cierto perfil diferenciado en los distintos centros. A este respecto, ya se ha anotado el perfil más filológico del grado en Lenguas y Literaturas Modernas, maior en chino de la UGR, pero también cabe mencionar la implementación del itinerario de árabe en la 
UAM, el de Corea en la US/UM, o la mención vinculada al área de las ciencias sociales del grado de EAO en la UAB.

\subsection{Estudios de lengua china o sobre China en grados del área de humanidades y ciencias sociales}

Las universidades españolas, generalmente en sus facultades de Filosofía y Letras, Filología, Traducción o de Comunicación, ofertan en sus planes de estudio asignaturas vinculadas al desarrollo de competencias en la lengua china. Así, en 7/22 grados en Lenguas Modernas y sus Literaturas ${ }^{3}$ que actualmente se ofertan en las universidades españolas es posible elegir el chino como segunda, tercera o cuarta lengua (tabla 4). En los grados de Traducción, en cambio, solo en 5/31 se oferta esta posibilidad en el plan de estudios (tabla 5).

TABLA 4

Presencia de la lengua china en los grados de Lenguas Modernas

\begin{tabular}{|l|c|}
\hline \multicolumn{1}{|c|}{ Universidad } & Créditos de lengua china \\
\hline Universidad Antonio de Nebrija & $>24$ ECTS \\
\hline Universidad Autónoma de Madrid & $>30$ ECTS \\
\hline Universidad Complutense de Madrid & $>30$ ECTS \\
\hline Universidad de Deusto & $>30$ ECTS \\
\hline Universidad de Granada & $>30$ ECTS \\
\hline Universidad de Las Palmas de Gran Canaria & $>12$ ECTS \\
\hline Universitat de València (Estudi General) & $>18$ ECTS \\
\hline
\end{tabular}

Fuente: elaboración propia.

\footnotetext{
${ }^{3}$ Hemos incluido bajo esta referencia también los grados denominados Lingüistica y Lenguas Aplicadas, Lenguas modernas y Traducción, Lenguas modernas y Traducción, Filología y Lenguas Aplicadas.
} 
TABLA 5

Presencia de la lengua china en los grados de Traducción e Interpretación

\begin{tabular}{|l|c|}
\hline \multicolumn{1}{|c|}{ Universidad } & N. ${ }^{o}$ máx. de créditos de lengua ofertados \\
\hline Universidad Autónoma de Barcelona & $>66$ ECTS \\
\hline Universidad Autónoma de Madrid & $>6$ ECTS \\
\hline Universidad de Alicante & $>24$ ECTS \\
\hline Universidad de Granada & $>60$ ECTS \\
\hline Universitat de València (Estudi General) & $>12$ ECTS \\
\hline
\end{tabular}

Fuente: elaboración propia.

En esta oferta académica se observan varios puntos en común, a saber:

Pese a que el total de créditos en relación con la enseñanza/aprendizaje de lengua china varía de una universidad a otra, y en consecuencia también lo hará el nivel de competencia alcanzado por los futuros egresados, la UAB y la UGR destacan, como ya lo hicieran antaño, como centros de referencia para el estudio de la lengua china, también en el grado de Traducción e Interpretación.

El chino se ha incluido habitualmente en los planes de estudio como lengua $\mathrm{C}$ o D, es decir, como tercera o cuarta lengua extranjera. Significativamente, ANECA (2004, pp. 34-35) señala que de una lengua $C$, además de no presuponerse conocimientos previos, solo se espera que el alumno desarrolle una competencia pasiva, y de una lengua D ningún nivel. Sin embargo, esta afirmación quizá sea matizable en determinados planes de estudio. Por ejemplo, en la guía docente 2019-20 de la asignatura Idioma y traducción C6 (chino), de $4 .^{\circ}$ curso del $1 .^{\text {er }}$ semestre del grado de Traducción de la UAB, los resultados de aprendizaje explicitan el objetivo de alcanzar un nivel B1.2. (MCRE) en la producción de textos escritos y un nivel A2.2. (MCRE) en la producción de textos orales.

El proyecto coordinado por Saquero (ANECA, 2006) reconocía su incapacidad para determinar de forma rigurosa y coherente la presencia de las áreas de conocimiento de las filologías en otras titulaciones, aunque en términos generales sí dejaba clara la exigua presencia de lo que llamaba "lenguas minoritarias", como, por ejemplo, el chino. Nuestros medios, mucho más precarios, posiblemente tampoco hubieran logrado dicha tarea. No obstante, a 
modo de muestra ilustrativa, a continuación sí se anota la situación existente en ciertos grados del área de las ciencias sociales y del área de humanidades, en los que, dada la actual situación sociopolítica y socioeconómica, las competencias en lengua y cultura china podrían ser de gran interés para sus egresados. A este respecto se comprobó la posibilidad de cursar alguna asignatura vinculada específicamente a China o al chino en los planes de estudios de los grados de Turismo (o Gestión Turística), Administración y Dirección de Empresas, Historia, Historia del Arte y Geografía en universidades en las que ya se ha implementado un grado, minor o área de conocimiento de Asia Oriental. Los resultados evidencian cómo la presencia de China o del chino en los planes de estudio es algo más que exigua, incluso cuando, en ocasiones, sí que se han implementado asignaturas con un marcado carácter regional, como, por ejemplo, Geografía y medio ambiente en África (OB, UV), Historia de los Estados Unidos (OB, UAM), Historia de América Contemporánea (OB, UAB), Historia antigua I: Historia del Próximo Oriente, Egipto y Grecia (OB, UGR). También es reseñable el hecho de que, aun cuando estas universidades tengan activadas asignaturas como Lenguas Extranjeras Aplicadas al Turismo (UAM), no se oferte el chino (tabla 6). ${ }^{4}$

TABLA 6

Presencia del chino o China en el plan de estudios de grados de humanidades $y$ ciencias sociales

\begin{tabular}{|l|c|c|c|c|c|c|}
\hline & Turismo & $A D E$ & Geo & HUM & $H^{a}$ & $H^{a}$ Arte \\
\hline USAL & No & No & No & No & No & No \\
\hline UV & No & No & No & - & No & No \\
\hline UGR & No & No & No & - & No & No \\
\hline UAM & No & No & No & - & No & Sí $^{5}$ \\
\hline UAB & Sí & No & No & No & No & Sí \\
\hline US/UM & No & No & No & - & No & No \\
\hline
\end{tabular}

Fuente: elaboración propia.

${ }^{4}$ Sin embargo, la UAB sí posibilita la formación en lengua china, tanto en la opción Segunda lengua extranjera II, Chino, como en la opción Tercera Lengua Extranjera I, Chino.

${ }^{5}$ Se incluye la asignatura de Arte de Asia Oriental (FB, 6 ECTS). 
Tal y como se mencionó al inicio de este texto, en las universidades españolas no existen departamentos de chino o China. En la mayor parte de los casos el desarrollo de competencias específicas sobre estas cuestiones se circunscribe al área de conocimiento de Asia-Oriental dependiente de alguno de los departamentos de las facultades de Letras y Filosofía, Filología, Traducción e Interpretación o Comunicación. Dado que en la Universidad española suele primar la organización intrafacultativa, dicha adscripción supone que habitualmente el alumnado procedente de titulaciones vinculadas a otras facultades tenga un difícil acceso a esta oferta docente, bien sea por la configuración de su plan de estudios, bien sea por la distribución horaria y espacial de las facultades. Así, no es de extrañar que, aunque en centros como los antes mencionados existan potenciales interesados en materias como, por ejemplo, Economía y Finanzas de Asia Oriental, Turismo y Flujos Migratorios en Asia Oriental o Geografía de Asia, su adscripción a facultades distintas o la ubicación en campus diferentes dificulta notablemente el acceso a estas, en el caso de que administrativamente sea posible.

\section{VALORACIONES FINALES}

El siglo Xxi ha supuesto un gran avance en lo que se refiere a la implementación de estudios sobre China o el chino en el ámbito universitario español, lo que ha permitido responder a la demanda creciente de profesionales con competencias en estas materias.

Un primer hecho reseñable es la implementación de grados vinculados al área de Asia Oriental con itinerarios o menciones específicas sobre China o el chino. Sin embargo, aunque se trata de una demanda creciente (y durante mucho tiempo ignorada), es todavía una lengua minoritaria en nuestro país, por tanto, parece muy pertinente la decisión de los responsables académicos de intentar diferenciar y singularizar su oferta.

La implantación de estos grados con itinerarios o menciones específicas sobre China o el Chino ha permitido incrementar de forma notable el conocimiento de la lengua y sociedad china de sus egresados. No obstante, dada la configuración interdisciplinar del plan de estudios de algunos de estos grados y la asimetría de la distancia interlingüística entre el español y el chino 
(López, 2008), es muy probable que los estudiantes necesiten completar su formación lingüística para desarrollar determinadas competencias profesionales. Los estudios en el nivel de posgrado o los convenios de intercambio son algunas de las posibilidades habilitadas por las universidades para atender dicha exigencia.

Dicho esto, y especialmente si se considera lo que parece será la configuración política, social y económica del s. XXI, queda por atender la necesidad (o el interés) que estudiantes y futuros profesionales de diferentes ámbitos tienen de formarse en cuestiones relativas a China y el chino. En este sentido, el estudio exploratorio realizado aquí ha ilustrado cómo, aun en las universidades en las que ya se encuentran habilitadas áreas de conocimiento y recursos humanos con competencias específicas en China o el chino, dicha formación apenas se contempla en el nivel de grado. Quizá sean necesarios más recursos humanos, quizá sea necesario promover la creación de departamentos interfacultativos de Asia Oriental, pero, por el momento, solo en el nivel de posgrado existe esa formación para, por ejemplo, futuros profesionales de la empresa, el turismo o las relaciones internacionales.

\section{BiBLIOGRAFÍA}

ANECA (2004). Libro Blanco. Título de Grado en Traducción e Interpretación. Recuperado de: http://www.aneca.es/var/media/150288/libroblanco traduc_def.pdf.

ANECA (2006). Libro blanco. Título de grado en estudios en el ámbito de la lengua, literatura, cultura y civilización. Recuperado de: http://www. aneca.es/var/media/150244/libroblanco_lengua_def.pdf.

Baltar, E. (1993). Filipinas en el contexto del sistema colonial español. Revista de estudios históricos 18, pp. 87-192.

Ministerio de Asuntos Exteriores (2002). Libro blanco sobre oferta y demanda de estudios asiáticos en España. Madrid: Ministerio de Asuntos Exteriores.

FisAC BAdELl, T. (2004). La formación sobre Asia-Pacífico en España. Anuario Asia Pacífico 1, pp. 401-406. 
López Álvarez, J. R. (1978). Los españoles, pioneros en la transmisión del pensamiento chino a Europa (Apuntes para una sinología española). $G a$ des 1, pp. 37-54.

López García, Á. (2008). Sobre el grado de cercanía a las lenguas de Asia Oriental: el caso del tagalo, en P. San Ginés (coord.), Nuevas perspectivas de investigación sobre Asia Pacífico (pp. 13-28). Recuperado de: http:// www.ugr.es/ feiap/ceiap2v2/ceiap/capitulos/capitulo01.pdf.

Lu, J. (2005). Enseñanza del español en China, en N. Ignatieva y V. Zamudio (eds.), Las lenguas extranjeras en un mundo cambiante (pp. 67-83). México: Universidad Nacional Autónoma de México.

Prosser van Der Riet, M. (2007). A study of the policy of promotion of minority languages in China (tesis doctoral). Recuperado de: http://hdl.handle. net/10803/10319.

Querol-Bataller, M. (2010). Estudios sobre la lengua china en España, en M. A. Montaner y M. Querol (coords.), Lenguas de Asia Oriental: Estudios Lingüísticos y Discursivos (pp. 43-88). Valencia: LynX, Panorámica de Estudios lingüísticos, Anexa 18.

Querol-Bataller, M. (2014). La especialidad de Español como carrera universitaria en China, MarcoELE, 18, Recuperado de: http://marcoele.com/ descargas/18/querol-espanol_en_china.pdf.

Rovira i Esteva, S. (2006). La lingüística no tiene quien le escriba ó 10 años de soledad. Condiciones para la investigación en lingüística china en España: historia reciente y perspectivas, en P. San Ginés (ed.), Foro español de investigación sobre Asia-Pacífico (FEIAP) (pp. 55-76). Granada, Universidad de Granada.

WAng, S. (2010). Chinese language education in the United States, en J. Chen, Ch. Wang y J. Cai (ed.), Teaching and learning Chinese: Issues and perspectives (pp. 3-32). UEA: IAP. 